\title{
Joint Access and Backhaul Power Consumption Optimization in Heterogeneous Mobile Broadband Networks
}

\author{
Georgios Kyriazis and Angelos Rouskas \\ Department of Digital Systems, University of Piraeus, 80, Karaoli \& Dimitriou Str, \\ Piraeus, GR-18534, Greece \\ E-mail: \{gkyriazi,arouskas\}@unipi.gr
}

Received 21 November 2016; Accepted 24 March 2017;

Publication 21 April 2017

\begin{abstract}
Capacity overprovisioning in mobile networks leads to underutilization of network resources and thus inefficient operation from a power consumption perspective. In addition, as operators constantly evolve their networks to denser heterogeneous layouts, with overlaying macrocellular base stations coexisting with numerous smaller cells, to cope with upcoming increase in peak mobile data traffic, the energy consumption of mobile networks will continue to grow in the next years. In this context, although backhaul connections can be responsible for tremendous power consumption, the corresponding study is usually neglected in most of the recent energy related literature. An approach for fast and low cost connectivity of dense microcellular sites is the deployment of wireless in-band backhaul links via existing macrocellular base stations. In this paper, we consider this wireless in-band backhaul approach, where access and backhaul links share the same frequency spectrum, and examine joint energy efficient resource optimization on both links. We introduce an iterative low-complexity polynomial backhaul-aware heuristic, which directs traffic to the most energy efficient network resources, and compare it against i) an exhaustive search method that provides optimum results,
\end{abstract}

Journal of Green Engineering, Vol. 6_4, 337-368.

doi: 10.13052/jge1904-4720.641

(c) 2017 River Publishers. All rights reserved. 
ii) a previously proposed reference algorithm, and iii) ordinary Full Operational Topology (FOT) network, where no energy aware technique is applied. From the results obtained, we conclude that our heuristic achieves high energy efficiency gains in all scenarios examined, while wireless in-band backhaul method proved a solid choice for this purpose.

Keywords: Energy efficiency, green communications, LTE, wireless in-band backhaul, heterogeneous mobile topology.

\section{Introduction}

The forecasts are predicting a tremendous increase in mobile data traffic in the next five years. Global mobile data traffic was increased by $74 \%$ in 2015 and reached 3.7 exabytes per month at the end of 2015, higher than 2.1 exabytes per month at the end of 2014 [1-2]. Nearly eightfold increase is expected for mobile data traffic, which will grow at a Compound Annual Growth Rate (CAGR) of 53\% between 2015 and 2020, reaching 30.6 exabytes per month by 2020 and the mobile data traffic per device will reach up to 22 gigabytes per month by 2021 [3]. With the current mobile data traffic growth rate, the current mobile networks capacity will soon be exhausted. Thus, the upcoming wireless communications systems have to achieve a thousand-fold capacity improvement goal. One of the main questions is how to accomplish this capacity goal without leading to an unacceptable increase in network power consumption, which in its turn raises crucial environmental concerns and yields increase in operational costs. The above challenges have attracted a lot of attention both by industry and academia, where green communications are continuously motivated to provide innovative solutions to respond to the high traffic demands in the most energy efficient way possible.

Denser heterogeneous mobile networks, consisting of overlaying large and small cells with same or different technologies are expected to alleviate traffic congestion and extend network's coverage and throughput capacity. Heterogeneous networks can support higher data volumes by reducing the distance between the transmitter and the receiver. In theory, we can effectively improve network capacity by reducing the cell size, since network capacity scales linearly with the cell density. Additionally, the lower distance among communication points results in lower path loss and in total network transmission power alleviation, thus increasing energy efficiency [4]. 


\subsection{Related Work and Trends}

Recent research in the literature reports that heterogeneous network topologies can greatly benefit the total network energy efficiency. Cells' size, density, and deployment strategies are optimized to improve the energy consumption in heterogeneous networks by over $96 \%$ in specific traffic scenarios [5-10]. Sleep/idle mode techniques, such as static or dynamic activation/deactivation of Base Stations (BSs) according to traffic load, latency and capacity variations, and optimal control of wake-up mechanisms can also yield great energy savings [11-13]. Cell breathing techniques applied on low network traffic conditions, by switching off any unnecessary BS and simultaneously expanding the coverage of some other BSs in order to guarantee coverage, can enhance energy efficiency [14-19]. Iterative minimal set covering algorithms to determine the minimum set of active BSs in heterogeneous network deployments under various Radio Access techniques (RATs) can reduce the energy spent during networks' daily operation [20-22].

What is neglected in the above works is that small cells operation can inflict a hefty traffic load to the backbone network [23-26] imposing also a considerable power burden to the system. Some researchers have pointed out its importance since backhaul (BH) consumption may vary from $30 \%$ to very high levels, which is comparable to the consumption levels of operating the macrocellular (macro) BSs [27-30]. Moreover, the backhaul impact on architecture deployment and operational costs also emphasizes its significance [31-32]. Thus, mobile networks evolution towards dense heterogeneous networks, with the presence of many smaller Access Points (APs) for providing the desired capacity, renders wireless backhaul worth considering within the scope of the energy-efficient design of heterogeneous networks [33].

\subsection{Approach and Outcomes}

In this paper, we consider wireless in-band backhaul solution to support ultra dense cellular environments. Wireless in-band backhaul is defined in 3GPP release 10 of LTE, as the BS-to-AP link which operates in the same carrier frequency as the AP-to-UE link [34]. The general idea with self-backhauling in mobile frequency bands is to reuse frequencies and radio interfaces/radio technology, normally used for the mobile access, also for the backhaul.

For in-band use, the backhaul and mobile end users will share the same radio interface and also share the available capacity on that radio interface [35]. An in-band full-duplex system presents a spectrum reuse scheme to wirelessly 
backhaul smaller cells with macro BSs without having to orthogonalize allocated spectrum between access and backhaul [36, 37].

Wireless in-band relays guarantee low CAPEX costs for wireless backhauling without the need for new spectrum, which is scarce in many countries. Additionally, wireless in-band backhaul solution enables operator to reuse a scarce resource which already possesses. Furthermore, in low-density rural areas, backhaul competes as the major candidate of the network operating expense [38]. Thus, wireless in-band relay/backhaul solutions can be used as low-cost alternatives for providing connectivity to remote cells covering mountainous or sparsely populated regions, or to temporary coverage cells when major events are being held or disasters strike. The same approach can also be effective for urban scenarios, where dense AP deployments would encounter installation difficulties, such as a large number of utility poles, cables etc. [39, 40]. Wireless in-band backhaul was also evaluated as candidate technique for throughput increase in massive MIMO systems [41]. Furthermore, the utilization of lower $\mathrm{GHz}$ band spectrum can overcome the Non-Line of Sight (NLOS) issues which other wireless backhaul solutions have to deal with.

The main culprit of power consumption in mobile telecommunication networks is the long-term use of macro BSs, whose operation results in high energy consumption on both transmission and auxiliary equipment components [42]. On the other hand, in the presence of wireless in-band backhauling, favouring the utilization of lower transmission power capable BSs only to minimize network's overall power consumption may not be the optimal approach to follow, since wireless in-band backhaul rests on macro BSs' resources for its operation. Therefore, without macro BSs and micro BSs joint optimization for access and backhaul links coverage, wireless network behaviour may be far from optimal from a power consumption perspective.

In our work, we investigate the joint optimization of backhaul and Access Links (AL) resource assignments taking into account power consumption in networks with wireless in-band backhaul, where the same carrier is utilized for both backhaul and access links. We first formulate the power consumption of heterogeneous networks as an optimization problem encapsulating wireless in-band backhaul transmissions. The problem falls into the category of Capacitated Facility Location Problems (CFLP), bounded by Single Sourcing constraint, which is NP-hard [43]. We then introduce a backhaul-aware energy efficient heuristic for minimizing the energy needed to operate these heterogeneous networks. To the best of our knowledge, similar works have been presented in [44-45]. Our approach utilizes the wireless in-band backhaul 
framework only and is differentiated from the approach in [44], where a dual connectivity backhaul scheme is used, including fixed and wireless backhauls. Additionally, two main contributions differentiate the current work from our previous work presented in [45]; the introduction of the mathematical formulation of the optimization problem and the proposal of an energy efficient heuristic to tackle energy consumption jointly on backhaul and access links.

The proposed energy efficient heuristic, namely the EneRgy Efficient Backhaul Oriented base Stations' (EREBOS) activation scheme, is a lowcomplexity polynomial heuristic which takes into account the wireless in-band backhaul connectivity when estimating the most efficient way to serve the corresponding network's traffic load, thus exploiting all available resources to serve both access and backhaul transmissions. We evaluated EREBOS in various traffic loads and its performance is compared against: $i)$ the optimum, derived by an exhaustive search method, ii) a reference algorithm (HTB), presented in [45], and iii) the ordinary Full Operational Topology (FOT) network, where no energy efficient technique is applied. The results indicate near optimum performance of EREBOS, outperforming the reference algorithm. EREBOS yielded quite satisfactory energy savings and increased useful payload transmissions per energy consumed, while guaranteeing Quality of Service (QoS) to the User Equipment (UEs). Concluding, the wireless in-band backhaul method proved as a solid backhaul solution in almost all scenarios examined.

The remainder of this paper is organized as follows. In Section 2, we present our system model and problem statement. In Section 3, we describe the energy efficient optimization scheme in detail. The performance evaluation is given in Section 4, and finally, the paper is concluded in Section 5.

\section{System Model and Problem Formulation}

Our system model consists of $J$ heterogeneous BSs and $I$ UEs, each one requesting a Guaranteed Bit Rate (GBR). In the context of this work, GBR defines the required QoS for every UE, served either by macro or micro BS. Wireless in-band backhaul solution is applied as the data transmission method from and to core network between higher transmission power capable BSs (macro) towards lower transmission power capable BSs (microcellular (micro), femtocells, etc.). Network mechanisms are utilized to associate UEs to the available BSs. For this association procedure, different approaches may be applied, depending on network operational requirements. Our network operational strategy aims on network operation with optimal power consumption. 
Therefore, power consumption should be kept to the minimum possible levels without affecting or with minimum effects on UEs' QoS.

More specifically, we study the forward channel of a multiple cell SingleInput Single-Output Orthogonal Frequency Division Multiple Access (SISO OFDMA) network, featuring Long Term Evolution (LTE) RAT. According to the LTE terminology $K$ frequency Resource Blocks (RBs) comprise the system bandwidth and we assume that the total transmit power of each BS is equally distributed among its RBs. Transmissions occur on a frame-byframe basis, where each frame consists of several OFDMA symbols. Inter-cell interference is also considered, as well as small scale fading and shadowing.

Inside this area, $I$ User Equipment (UEs) are uniformly and randomly distributed, each one demanding access and requesting a GBR. Each UE is served by a specific cell with a set of RBs (corresponding to a specific bitrate) assigned to it to satisfy its traffic request. We assign each block/frame to one forward access or backhaul transmission at most to preserve time and frequency orthogonality and eliminate intra-cell interference. However, the UE suffers from inter-cell interference by neighboring cells that also utilize the same RBs. For the estimation of inter-cell interference signals, we relied on the WINNER II model [46] soft inter-cell interference cancellation method, which is based on the use of more reliable soft-values provided by the decoding process. This approach adds complexity to the system, since a channel decoding step has to be performed for each interferer [47]. Nevertheless, a different number of RBs may be assigned to each transmission in the same frame. We assume a reuse factor equal to 1 , so each cell (macro or micro) can utilize all the available RBs. Finally, several downlink resources are devoted to signaling/reference transmissions, hence only a portion of the system power is dedicated to serving useful payload.

We assume that macro BSs are connected to the core network via high capacity reliable connections, e.g. fiber or fixed wireless connection. Wireless in-band BH framework is deployed as the data transmission method between macro BSs and operational micro BSs. Macro BS radio resources, e.g. macro subchannels, are utilized at the wireless in-band backhaul links for transmitting the requested traffic load to the micro BS from the core network, thus the same carrier is utilized for both backhaul and access links, as shown in Figure 1. When the UE is served by a macro BS, data are routed from the Mobility Management Entity (MME) / Serving Gateway (S-GW) core network nodes via the high capacity connection to the macro BS and then wirelessly transmitted through the macro BS access link towards the UE. On the other case, where UE is served by a micro BS, data are routed from MME/S-GW 


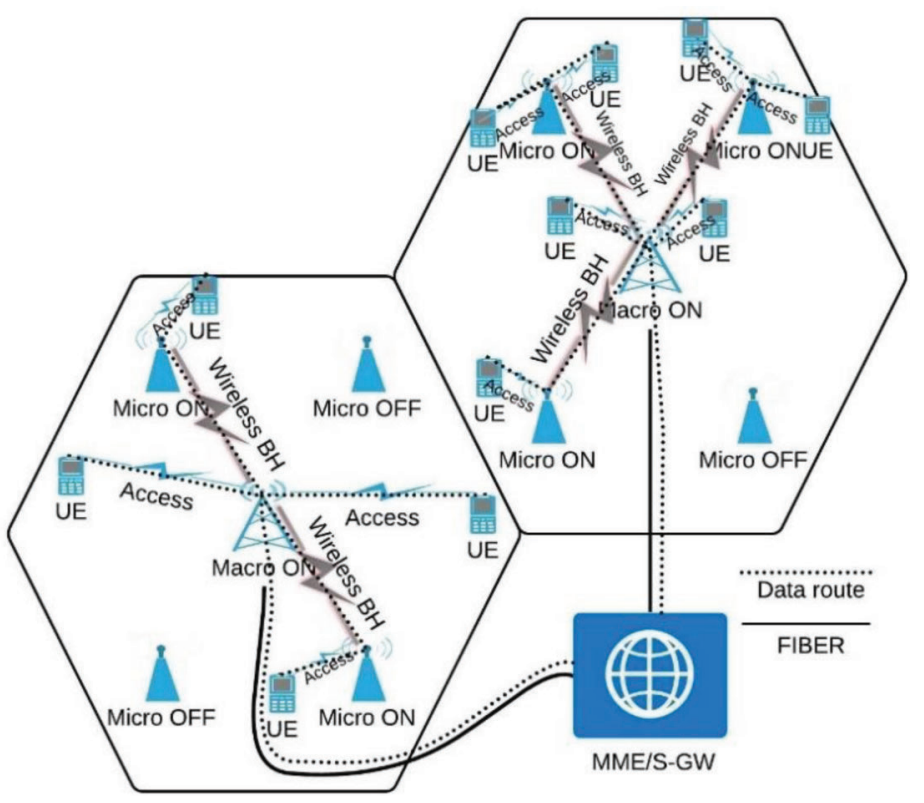

Figure 1 System model.

node via a high capacity connection to macro BS, then through the wireless in-band backhaul link to the micro BS and finally through the micro BS access link towards the UE.

We assume the existence of a perfect controller at the macro BS, which can dynamically distribute data, received from the core network via the high capacity connection, to operational micro BS and UEs that are simultaneously served, with negligible impacts on delay and interference. Last but not least, smaller BS antenna characteristics of micro BSs, e.g. antenna sensitivity, gain, etc., allows high useful payload throughputs per resource block via the air interface of macro BSs, which can be also available to much longer distances compared to mobile UEs' capabilities.

We now may break down our problem statement as follows:

- The heterogeneous network consists of $n, n=1,2, \ldots, N$, tiers each one consisting of identically configured BSs sets. Assuming that $n=1$ indicates the macro BSs tier, let $J_{1}$ be the number of the macro BSs and $D_{1}=\left\{j \mid j=1, \ldots, J_{1}\right\}$ be the set of these $J_{1}$ macro BSs. The second tier set of $J_{2}$ BSs is $D_{2}=\left\{j \mid j=J_{1}+1, \ldots, J_{1}+J_{2}\right\}$, the third tier set of $J_{3}$ BSs is $D_{3}=\left\{j \mid j=J_{1}+J_{2}+1, \ldots, J_{1}+J_{2}+J_{3}\right\}$ and so on, 
where $J_{2}$ and $J_{3}$ denote the numbers of micro and femtocell BSs respectively. Similarly, the number of the available heterogeneous BSs in a n-tier network is $J=J_{1}+J_{2}+J_{3}+\cdots+J_{N}$ and the $N$-th tier set of BSs is $D_{N}=\left\{j \mid j=J-J_{N}+1, \ldots, J\right\}$. For instance, in the above example we have $n=3$ tiers of BSs, where $n=1$ denotes the macro BSs tier, $n=2$ denotes the micro BSs tier and $n=3$ denotes the femtocell BSs tier.

- Each BS may occupy one out of two possible states, namely operational $(\mathrm{ON})$ and non-operational (OFF). Each operational BS exhibits (a) a fixed power consumption cost $f_{j}$ derived from its auxiliary equipment utilization, and (b) a variable cost $v_{i j k}$, which is inflicted by the utilization of subchannel $k$ when UE $i$ is served by the access link of BS $j$ with achievable bitrate $b_{i j k}^{(A L)}$, due to the extra energy consumed by the equivalent Power Amplifier (PA), where $i=1, \ldots, I, j=1, \ldots, j, k=$ $1, \ldots, K$. It is easy to see that operational BSs of disparate BSs' tiers have both variable and fixed power costs different, due to the different nature of their components and equipment.

- Each UE $i, i=1, \ldots, I$, when served by BS $j$ acquires an Access BitRate (ABR), whose lower limit is defined by GBR $g_{i}$, therefore a successful association of UE $i$ to BS $j$ ensures the satisfaction of its GBR $g_{i}$ demand.

- Resources of operational macro BS $j$ may be utilized to transmit the Backhaul BitRate (BBR) that serves the sum of ABRs of some nonmacro operational BS $m$, where $j=1, \ldots, J_{1}, m=j_{1}+1, \ldots, J$. Such BH transmissions inflict an additional $u_{m j k}$ variable power cost to the network, due to the utilization of subchannel $k$ of macro BS $j$ with achievable bitrate $b_{m j k}^{(B H)}$, for transmitting ABR data of BS $m$ via wireless in-band BH transmissions, $k=1, \ldots, K, j=1, \ldots, J_{1}, m=J_{1}+1, \ldots, J$.

As aforementioned, our goal is to optimize the operation of a heterogeneous wireless broadband telecommunication network with respect to energy consumption, while QoS requirements are preserved. Thus, in our approach, the problem at hand is to find the most appropriate subset of the existing BSs, which is adequate to serve the requested traffic demands of the UEs without compromising their QoS requirements and with minimum possible power consumption.

The optimization problem can now be formulated as follows:

$$
\min \left\{\sum_{i=1}^{I} \sum_{j=1}^{J} \sum_{k=1}^{K} v_{i j k} \cdot x_{i j k}+\sum_{m=J_{1}+1}^{J} \sum_{j=1}^{J_{1}} \sum_{k=1}^{K} u_{m j k} \cdot z_{m j k}+\sum_{j=1}^{I} f_{j} \cdot y_{j}\right\}
$$


Subject to:

$$
\begin{gathered}
\sum_{k=1}^{K}\left(x_{i j k}+z_{m j k}\right) \leq 1, i=1, \ldots, I, j=1, \ldots, J_{1}, m=J_{1}+1, \ldots, J \\
\sum_{k=1}^{K} x_{i j k} \leq 1, i=1, \ldots, I, j=J_{1}+1, \ldots, J \\
\sum_{j=1}^{J} x_{i j}=1, i=1, \ldots, I \\
\sum_{j=1}^{J_{1}} z_{m j}=1, m=J_{1}+1, \ldots, J \\
\sum_{j=1}^{J} \sum_{k=1}^{K} x_{i j k} . b_{i j k}^{(A L)} \geq g_{i}, i=1, \ldots, I \\
\left(\sum_{m=J_{1}+1}^{J} \sum_{j=1}^{J_{1}} \sum_{k=1}^{K} z_{m j k} \cdot b_{m j k}^{(B H)}\right)-\left(\sum_{i=1}^{I} \sum_{j=J_{1}+1}^{J} \sum_{k=1}^{K} x_{i j k} \cdot b_{i j k}^{(A L)}\right) \geq 0
\end{gathered}
$$

Where the following binary decision variables are introduced:

$$
x_{i j k}=\left\{\begin{array}{l}
1, \text { if channel } k \text { of } \mathrm{BS} j \text { is assigned to user } i \\
0, \text { otherwise }
\end{array}\right.
$$

which indicates whether channel $k$ of $\mathrm{BS} j$ is assigned to UE $i$,

$$
x_{i j}=\left\{\begin{array}{l}
1, \text { if } \sum_{k=1}^{K} x_{i j k}>0, i=1, \ldots, I, j=1, \ldots, J \\
0, \text { otherwise }
\end{array}\right.
$$

Which indicates whether BS $j$ serves UE $i$,

$z_{m j k}=\left\{\begin{array}{l}1, \text { if channel } k \text { of } \mathrm{BS} j \text { is assigned to serve } \mathrm{BH} \text { transmissions to } \mathrm{BS} m \\ 0, \text { otherwise }\end{array}\right.$

which indicates whether channal $k$ of $\mathrm{BS} j$ is assigned to $\mathrm{BS} m$ for wireless in-band $\mathrm{BH}$ transmissions,

$$
z_{m j}=\left\{\begin{array}{l}
1, \text { if } \sum_{k=1}^{K} z_{m j k}>0, m=J_{1}+1, \ldots, J, j=1, \ldots, J_{1} \\
0, \text { otherwise }
\end{array}\right.
$$


which indicates whether a macro BS $j, j=1, \ldots, J_{1}$, serves BBR transmission,

$$
y_{j}=\left\{\begin{array}{l}
1, \text { if } \sum_{i=1}^{I} x_{i j}>0, j=1, \ldots, J \\
1, \sum_{m=J_{1}+1}^{J} z_{m j}>0, j=1, \ldots, J_{1} \\
0, \text { otherwise }
\end{array}\right.
$$

which indicates that fixed power cost is inflicted: (a) when $\mathrm{BS} j, j=1, \ldots, J$, is operational to serve $\mathrm{ABR}$ transmissions, or (b) when a macro $\mathrm{BS} j, j=1, \ldots$, $J_{1}$, is operational to serve BBR transmissions.

The objective function presented in (1) aims at the minimization of the energy consumption of the network, by minimizing the sum of the variable and fixed energy costs inflicted by the utilization of certain BSs and the corresponding subchannels. Constraints (2) impose that each channel of every macro BS is assigned as an access link to one UE or as a backhaul link to one non macro BS at most. Similarly, constraints (3) impose that each channel of every non macro BS is assigned to one UE at most. Constraints (4) in combination with (8) and (9) impose that each UE is served by one and only one BS, while constraints (5) in combination with (10) and (11) impose that the backhaul of each non macro BS is served by one and only one macro BS. Constraints (6) and (7) represent the demand constraints. Constraints (6) impose that every BS will transmit at least each UE's minimum requested demand $g_{i}$. Finally, constraint (7) impose that the sum of BBRs of all macro BSs will support the sum of ABRs of all low transmission power BSs.

This problem belongs to the class of Capacitated Facility Location Problems (CFLPs), restricted by a Single Sourcing (SS) constraint. It is a capacitated problem since resources of each BS are limited. It is a facility location problem because the optimal placement of BSs aims at the minimization of the fixed power consumption (fixed costs) and the required RF transmission power (transportation costs). Finally, the Single Sourcing constraint is applied since each UE may be served by one and only one BS and each micro BS gets its backhaul transmission from one macro BS only. CFLP has been studied in [43], where, among others, heuristic and Linear Programming (LP) methods have been proposed for obtaining a feasible solution. Nevertheless, these methods are computationally complex and their implementation regarding the management of a telecommunication system, where decisions have to be both reliable and prompt, is not applicable. In the following section, we introduce a greedy heuristic algorithm to tackle this problem in a computational efficient way. 


\section{Proposed Algorithm}

The EneRgy Efficient Backhaul Oriented base Stations' (EREBOS) activation scheme receives as inputs a set of BSs with different power transmission capabilities at predefined locations and a set of UEs requesting a GBR. EREBOS is executed for certain traffic demand snapshots and achieves the minimization of network power consumption by toggling the necessary BSs to operational ('ON') state. Priority is given to BSs with lower power transmission capabilities which generally consume less energy and are capable of serving the UEs' traffic load while preserving the QoS constraints. In addition, EREBOS considers and evaluates backhaul links requirements towards joint access and backhaul power consumption optimization.

Although the algorithm can generally be extended up to n-tiers heterogeneous network topologies, in the context of this work we consider a two-layer heterogeneous network topology which consists of $J_{1}$ macro high power transmission capable BSs and $J_{2}$ micro low power transmission capable BSs sets. In addition, wireless in-band BH transmissions occur among macro towards micro BSs, only when micro BSs are operational. Each association of a micro to macro BSs for backhaul transmissions, consists a BH pair.

Intuitively, reducing macro BSs' resources utilization or substituting their operation by smaller scale BSs, whenever and wherever this is feasible, may probably lead to significant power consumption alleviation to network's operation. To this point, EREBOS's philosophy is based on utilization of the smaller scale (in our scenario the micro) BSs first for serving network's traffic load while utilizing macro BSs only when it is necessary, e.g. for BH transmissions or for serving UE which micro BSs cannot serve. Among the possible BS-UE pairs, EREBOS finds which BSs can serve the available set of UEs. When not all UEs traffic requirements and/or BH transmissions can be served by the available set of BSs, GBR requirement $g_{i}$ is relaxed by reducing GBR by some step value and EREBOS recalculates possible BS-UE pairs and proceeds its calculations from the beginning. Figure 2 depicts a high level formal flowchart of the proposed algorithm. A detailed description of the EREBOS's discrete steps follows.

A list of registered UEs and available BSs is created. We denote by $S_{M}^{(O N)}$ and $S_{m}^{(O N)}$ the sets of operational macro and micro BS and initialise these sets as $S_{M}^{(O N)}=S_{m}^{(O N)}=\varnothing$.

Step i. We calculate the feasible throughput $b_{i j k}^{(A L)}$ of every subchannel $k$ of BS $j$ to every UE $i, j \in D_{1} \cup D 2, i=1, \ldots, I, k=1, \ldots, K$, in a worst-case fashion, by setting all BSs to 'ON' state at this step. 


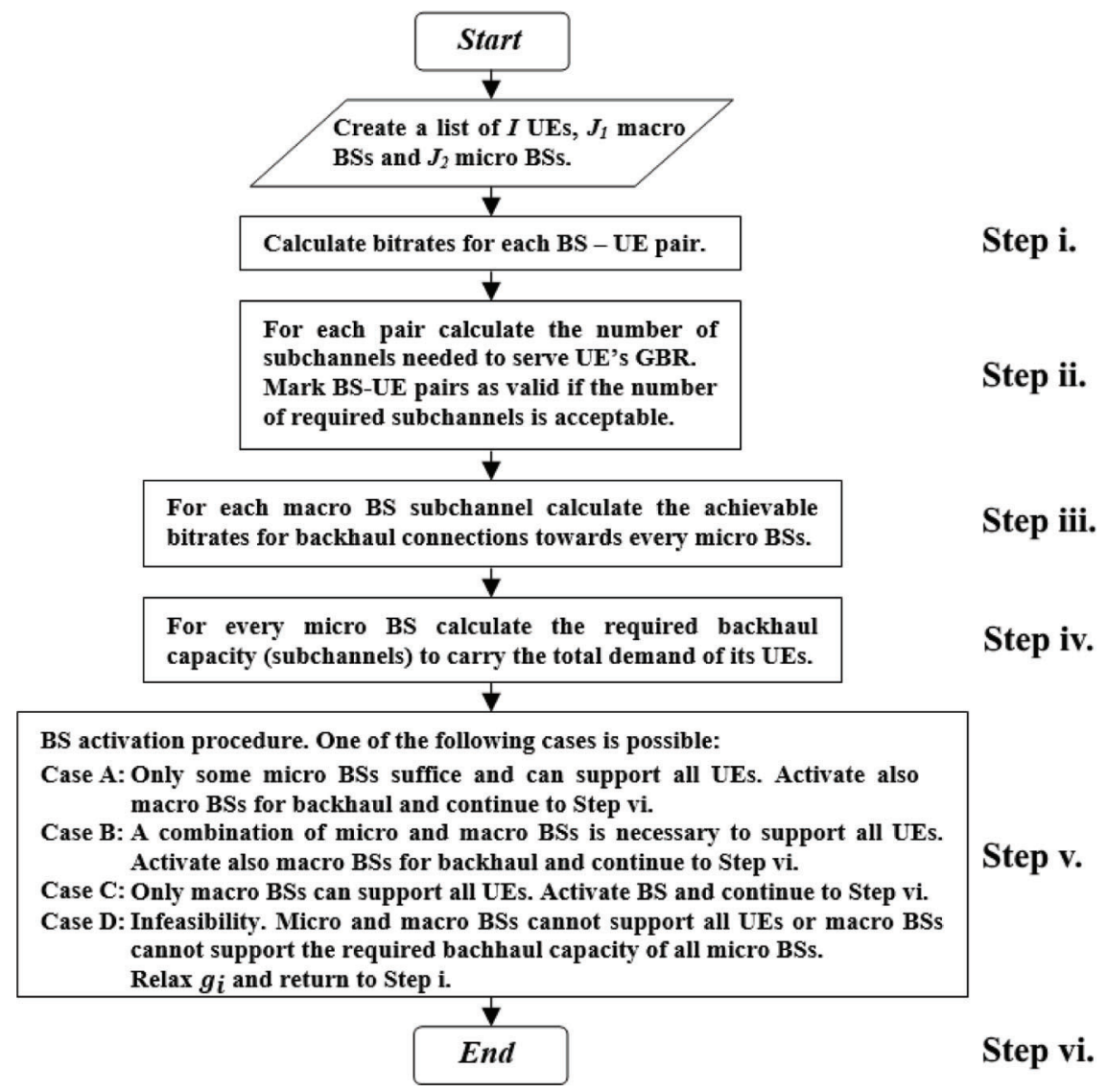

Figure 2 Flowchart of EREBOS algorithm.

This information is derived from the Channel State Information (CSI) blocks exchanged between the users and the network. Channel information per resource block is converted through the Shannon's capacity formula to Bit Rate (BR) per resource block (subchannel) and thus each UE roughly estimates the QoS which could be provided by the available BSs.

Step ii. If a BS is able to fulfill the QoS requirements of a UE, this BS-UE pair is marked as being valid. We evaluate the resources' assignment for every BS-UE valid pair as follows: if the number of resources needed exceeds a predefined threshold of the total number of subchannels of each BS, then we consider the corresponding BS-UE association 
pair as invalid. At this stage, each UE may participate in more than one pairings, although during the actual radio resource assignment stage the single source constraint is respected. Finally, the achievable throughput of all valid BS-UE pairs are stored in a two-dimensional matrix $M_{A B, t}$. The resources needed for each valid BS-UE pair association are stored in a two-dimensional matrix $M_{A B, r}$.

Step iii. We calculate the feasible throughput $b_{m j k}^{(B H)}$ of every subchannel $k$ of BS $j$ towards every BS $m, j \in D_{1}, m \in D_{2}, k=1, \ldots, K$ and store the results inside a three-dimensional matrix $M_{B B, t}$ matrix. Therefore, the $M_{B B, t}$ matrix contains the feasible throughput per subchannel $k$ of every macro BS when used for BBR transmissions towards each micro BS.

Step iv. We calculate the wireless in-band BH resources needed for every micro BS $m$ as follows. For every micro BS $m$ we calculate the total throughput of all UEs it may serve, which corresponds to the sum of its ABRs assuming that all its BS-UE pairs are enabled. This ABR sum must be transmitted to $\mathrm{BS} m$ via wireless in-band $\mathrm{BH}$ transmissions occupying resources from some macro BS $j, j \in D_{1}$. The number of resources needed for BH transmissions towards every micro BS $m$ are stored in a two-dimensional matrix $M_{B B, r}$. After this procedure, we revert all BSs' operational state to the state they had at the end of Step i, thus all BSs are set again to 'OFF' state.

Step v. BS Activation Step: EREBOS favours utilization of the smaller scale micro BSs first for serving network's traffic load while utilizing macro BSs only when it is necessary, e.g. for BH transmissions or for serving UEs which micro BSs cannot serve. The available macro BSs are placed inside $S_{M}$ list and the available micro BSs are placed inside $S_{m}$ list. We denote by $S_{m}^{(C)}\left(S_{M}^{(C)}\right)$ the list of micro (macro) BSs that have at least one valid BS-UE pair in $M_{A B, t}$ matrix and thus are candidates for being operational at the end of the algorithm. The following cases exist:

A: Only some micro BSs suffice and can support all UEs

$B$ : A combination of micro and macro BSs is necessary to support all UEs

$C$ : Only macro BSs can support all UEs

$D$ : Micro and macro BSs cannot support all UEs or macro BSs cannot support the sum of ABRs of all micro BSs. The situation is considered as infeasible. 
Step v.(A): When only micro BSs can support all the traffic load, we first sort $S_{m}^{(C)}$ in descending order of the number of UEs they can serve and set the first BS of that sorted list to operational 'ON' state. Ties are solved by selecting that BS which utilizes the fewer resources (e.g. fewer number of subchannels) and sub-ties by selecting the BS which can achieve the higher average throughput towards UEs served. The activated micro BS is moved from $S_{m}^{(C)}$ to $S_{m}^{(O N)}$ list and we exclude the UEs, with valid pairs to this micro BS, from further investigation. Then EREBOS proceeds with the activation of another micro BS the $S_{m}^{(C)}$ list, by parsing the list downwards and selecting the next micro BS to move to $S_{m}^{(O N)}$. This procedure is repeated until all UEs are served from micro BSs.

Since macro BSs are mandatory for serving the $\mathrm{BH}$ transmissions and none has been activated yet, the procedure proceeds as follows:

- we locate the operational micro BS which can serve the fewer UEs, ties are solved by selecting the one which utilizes the most resources, sub-ties by selecting the one which achieves the lower average feasible throughput towards the assigned UEs, and we set it to non-operational state and remove it from $S_{M}^{(O N)}$, and search for a macro BSs to serve the UEs which are no longer served after the deactivation of this micro BS. This macro BS is set to ' $\mathrm{ON}$ ' state, moved to $S_{M}^{(O N)}$ list and removed from $S_{M}^{(C)}$ if previously placed.

- The already operational macro BS is assigned to serve some of the already activated micro BSs' ABRs whose BH transmissions are not yet served.

- Finally, if some micro BSs' ABRs cannot be served by the already operational macro BS, a new macro BS is utilized, which will not serve any AL links but only BH transmissions. This macro BS is chosen based on the higher number of the $\mathrm{BH}$ transmissions it can serve. Ties are resolved by selecting 
the one which needs fewer resources to serve these BH transmissions and sub-ties based on the higher average feasible throughput it may achieve again towards these $\mathrm{BH}$ transmissions. This macro $\mathrm{BS}$ is set to 'ON' state, moved to $S_{M}^{(O N)}$ list and removed from $S_{M}^{(C)}$ list, if previously placed. This procedure is repeated until all micro BSs ABRs are served by $\mathrm{BH}$ transmissions.

Goto Step vi.

Step v.(B): When all UEs can be served only by a set of micro and macro BSs, the steps to yield $S_{m}^{(O N)}$ are similar to the steps described in Step v.(A). However, when selecting a macro BS to move to $S_{M}^{(O N)}$ the following differences apply:

- No already activated micro BS is deactivated.

- Macro BS selection criterion is based on the number of most unassigned UEs that macro BS can support. Ties are resolved by selecting the macro $\mathrm{BS}$ which can participate in the most $\mathrm{BH}$ pairs with some of the micro BSs in $S_{m}^{(O N)}$, sub-ties are resolved by selecting the one utilizing the fewer resources for $\mathrm{AL}$ and $\mathrm{BH}$ transmissions.

- Subsequently, already operational macro BSs are evaluated first on whether they can serve BH transmissions towards alreadyoperational micro BSs. From the already operational macro BSs, weselect the one whose $\mathrm{BH}$ transmissions can serve most of the operational micro BSs' ABRs. Ties are resolved by selecting the macro BS with the fewer available resources, sub-ties by selecting the one which achieves the higher average BBR throughput towards the micro BSs it may serve. The above procedure is repeated until all operational micro BSs become members of valid BH pairs.

In case, where some micro BSs' ABR cannot be served by the already operational macro BS, a new macro BS is utilized, which now will not serve 
any access links but only $\mathrm{BH}$ transmissions. The procedure is similar to the one at the last bullet in Step v.(A).

Goto Step vi.

Step v.(C): When all traffic can be served by macro BSs only, we sort $S_{M}^{(C)}$ in descending order of the number of UEs they can serve and set to operational ' $O N$ ' state the first BS. Ties are resolved by selecting the macro BS which utilizes fewer resources, sub-ties by choosing the one which achieves higher average feasible throughput towards the UEs it may serve. The activated macro BS is moved from $S_{M}^{(C)}$ to $S_{M}^{(O N)}$ list. The UEs assigned to this macro BSs are excluded from further investigation. The same procedure is repeated until all UEs are being served.

Goto Step vi.

Step v.(D): We record this infeasible solution and we proceed to GBR requirement $g_{i}$ relaxation, by reducing GBR by some step value (e.g. $1 \mathrm{kbps}$ ), and EREBOS procedure restarts its calculations from Step ii. In case where GBR's new value is lower than a predefined minimum acceptable GBR threshold (e.g. $100 \mathrm{kbps}$ ), this means that current network topology cannot serve the present traffic load requirements.

Step vi. The algorithm terminates either by successful assignment of all UEs to some BSs and all operational micro BSs ABR to BH pairs or by detecting infeasibility.

The proposed algorithm exhibits low polynomial-order complexity since it involves only sorting, searching and comparison operations among system elements and derivatives (BSs types, UEs, subchannels, and throughput). Step i of the algorithm requires $O(J \cdot K)$ time, since there are $J$ BSs with $K$ subchannels at most to examine. At Step iii up to $J_{1} \cdot J_{2}$ combinations can be executed, because the maximum number of macro (micro) BSs is $J_{1}\left(J_{2}\right)$, which takes $O\left(J_{1} \cdot J_{2}\right)$ time. Step iv requires $O\left(J_{2}\right)$ time since at most $J_{2}$ BSs will be parsed. At Steps v. (A-D) up to $J_{1} \cdot J_{2} \cdot K$ values are sorted which requires $O\left(J_{1} \cdot J_{2} \cdot K \cdot \log \left(J_{1} \cdot J_{2} \cdot K\right)\right.$ time. Finally, there are 
$I$ UEs and $J_{2}$ BSs to associate at most, thus the overall time complexity is $O\left(J_{1} \cdot J_{2}^{2} \cdot I \cdot K \cdot \log \left(J_{1} \cdot J_{2} \cdot k\right)\right)$. The complexity of the proposed greedy heuristic is polynomial and depends on the numbers of macro BSs, micro BSs, UEs and subchannels.

\section{Simulation Results}

The performance of the proposed algorithms was evaluated using a systemlevel simulation tool developed in MATLAB R2015a (8.5.0.197613 64-bit) on a desktop pc running Windows 10, Version 1607 (OS Build 14393.693), equipped with an intel $17-4790 \mathrm{k}$ processor matched with dual channel DDR3-2400 MHz RAM, loaded with basic daily activities. The WINNER propagation model is adopted incorporating LOS, as well as NLOS conditions [46]. All the attributes of the network were adjustable, while the sessions were statically created at the beginning of each simulation run. Different traffic load conditions were examined, by differentiating the number of UEs and the GBR of each UE in each run. EREBOS was evaluated in terms of energy efficiency against the optimum bound, derived by an exhaustive search method, and against a reference algorithm, namely the HTB presented in [45]. HTB algorithm receives as input from the HTWEAK algorithm [21] the optimal set of BSs that suffice to serve the present traffic load of the network and initiates the Backhaul Links Assignment procedure (BHLA) for yielding the macro BSs, able to carry the wireless in-band BH transmissions. Thus, HTB also uses wireless in-band backhaul technology, but performs assignment of UEs and $\mathrm{BH}$ associations sequentially, unlike EREBOS that jointly optimizes UE assignments and $\mathrm{BH}$ associations.

All numerical results are normalized against FOT, where all BSs are switched ON, while all metrics have been calculated by averaging $L=200$ results per scenario. A dense urban environment was examined, considering the network coverage area of a $1 \times 1 \mathrm{Km}^{2}$, where $J_{M}=9$ macro and $J_{m}=16$ micro BS locations are preconfigured in a grid, as shown in Figure 3.

Furthermore, $I=5,15,30,50$ UEs have been randomly and uniformly distributed within this region and each UE $i$ requested GBR $g_{i}=500,750$ or $1000 \mathrm{kbps}$. Bitrates are calculated in the following using Shannon's formula, based on a worst-case transmission scenario. Table 1 summarizes the values of the main parameters of our simulation model. 


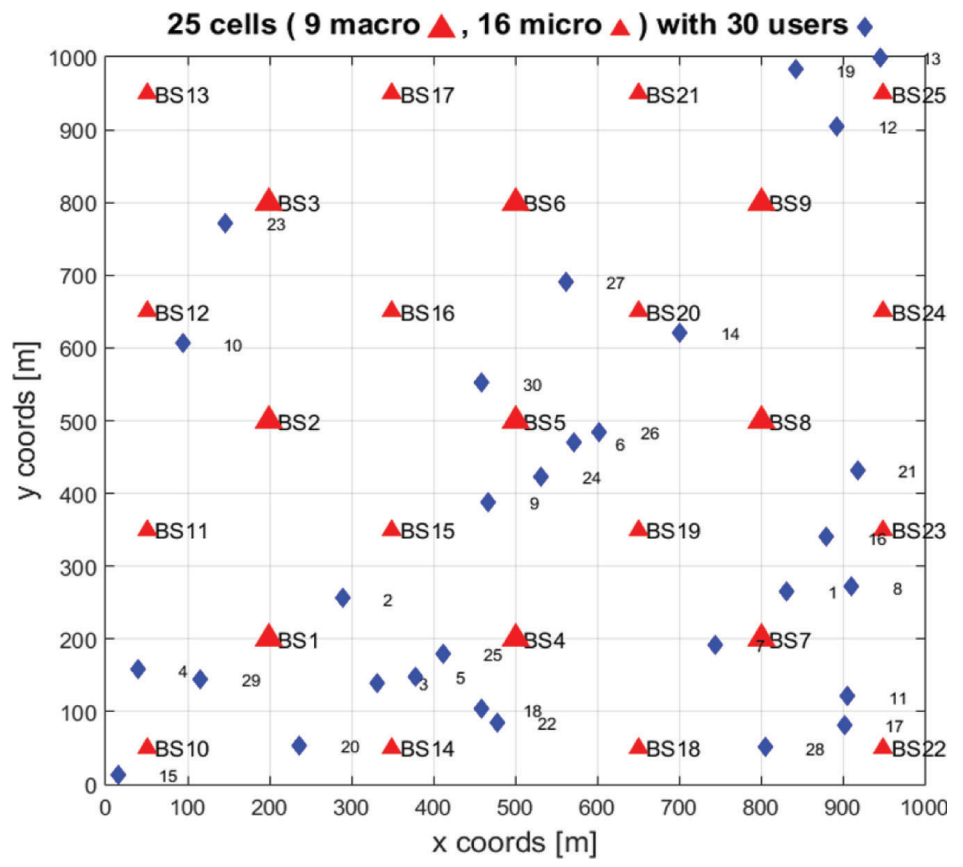

Figure 3 The network topology used in our simulations. Big (small) red triangles indicate macro (micro) BSs, blue diamonds represent UEs.

Table 1 Main parameters of our simulation model

\begin{tabular}{|c|c|c|c|}
\hline Parameter & \multicolumn{3}{|l|}{ Value } \\
\hline Number of resource blocks & \multicolumn{3}{|l|}{25} \\
\hline Bandwidth & \multicolumn{3}{|l|}{$5 \mathrm{MHz}$} \\
\hline Distribution of UEs & \multicolumn{3}{|l|}{ Uniform } \\
\hline GBR & \multicolumn{3}{|c|}{$\begin{array}{l}\text { Three simulation campaigns: } 500,750 \\
\text { and } 1000 \mathrm{Kbps}\end{array}$} \\
\hline Snapshots per scenario & \multicolumn{3}{|l|}{200} \\
\hline RAT & \multicolumn{3}{|l|}{ LTE } \\
\hline Propagation model (RAN) & \multicolumn{3}{|c|}{ C3 (B2) bad urban - WINNER II model [46] } \\
\hline Propagation model (BH) & \multicolumn{3}{|c|}{ Taken from 3GPP TR 36.814 V9.0.0 specification [49] } \\
\hline \multirow{4}{*}{$\begin{array}{l}\text { Typical power } \\
\text { consumption of macro } \\
\text { (micro) BSs }[42,48]\end{array}$} & Power Amplifier & $\sim 50-80 \%$ & $\sim 1200$ (145) Watts \\
\hline & Air conditioning & $\sim 10-25 \%$ & $\sim 300$ (0) Watts \\
\hline & $\begin{array}{l}\text { Signal processing } \\
\text { (analog/digital) }\end{array}$ & $\sim 5-15 \%$ & 200 (35) Watts \\
\hline & Power supply & $\sim 5-10 \%$ & $\sim 100$ (15) Watts \\
\hline & Original topology & \multicolumn{2}{|c|}{ Smaller topology } \\
\hline Number of BSs & 9 macro - 16 micro & \multicolumn{2}{|c|}{3 macro -8 micro } \\
\hline Number of UEs & $\{5,15,30,50\}$ & \multicolumn{2}{|c|}{$2,5,10,15$} \\
\hline
\end{tabular}




\subsection{Performance Comparison with the Optimal Results in Smaller Topology}

Additionally, we compared the results with respect to the optimum bound on a smaller network topology, consisting of $J_{M}=3$ macro and $J_{m}=8$ micro preconfigured in a $0.4 \times 1 \mathrm{Km}^{2}$ reference area as shown in Figure 4. The optimal results were acquired per snapshot by means of an exhaustive search, where all possible solutions were investigated and the most energy efficient one was selected as the optimum, for $I=2,5,10,15$ UEs and GBR $g_{i}=500 \mathrm{kbps}$. The use of this reduced reference area and traffic load was necessary because of the exponential computational complexity of the exhaustive search method.

As shown in Figure 5, EREBOS achieves near optimal average power consumption performance, with an average percentage increase of $\sim 7 \%$ compared to the optimum which is calculated by utilizing the exhaustive search method. Furthermore, EREBOS performs better than the reference HTB algorithm which exhibits an average $11.3 \%$ increase when compared to the optimal results. At the lowest traffic load with 2 UEs and GBR at $500 \mathrm{kbps}$ in the scaled down topology scenario, EREBOS algorithm achieves optimal energy efficiency, where a marginal increase of $0.53 \%$ in the average power consumption performance compared to the optimum is recorded. At the same load EREBOS outperforms the reference algorithm, which achieves an 8,4\% increase from the optimum.

\subsection{EREBOS and HTB Power Consumption against the FOT Consumption}

The normalized average power consumption for the sum of the network's traffic load snapshots is presented in Figure 6. The shown metrics are compared against a Fully Operational Topology (FOT) which is simply a scenario where

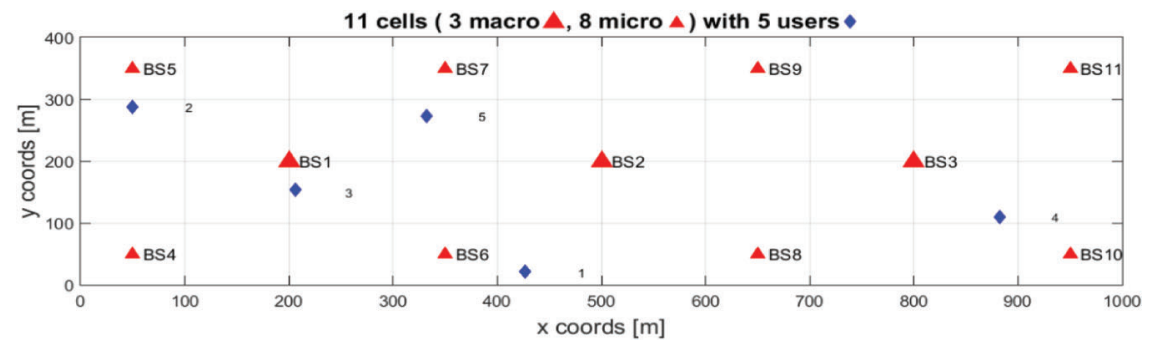

Figure 4 The smaller network topology used in our simulations to estimate EREBOS's performance compared to optimum. Big (small) red triangles indicate macro (micro) BSs, blue diamonds represent UEs. 


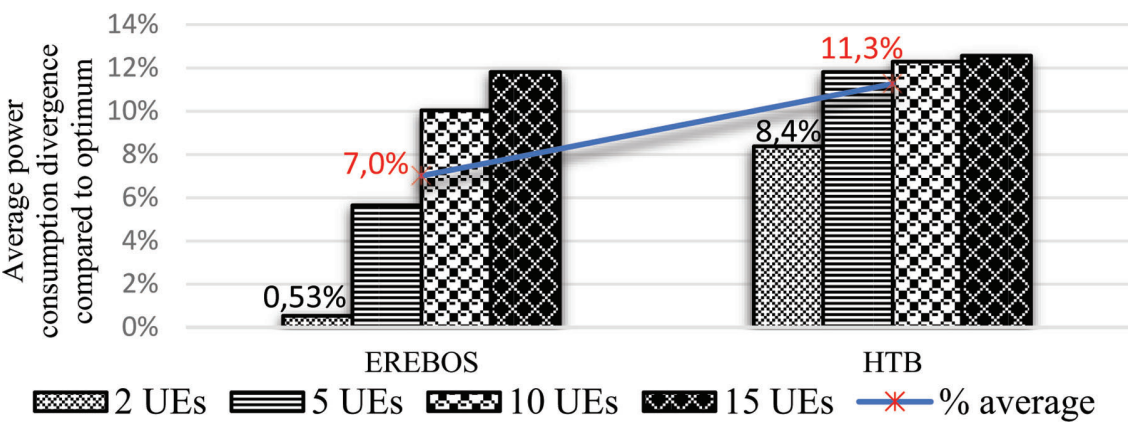

Figure 5 Performance of EREBOS and HTB algorithms expressed as power consumption percentage increase compared to the optimum results for traffic loads of 2, 5, 10 and 15 UEs, GBR of $500 \mathrm{kbps}$, evaluated in the scaled-down network topology depicted in Figure 4.

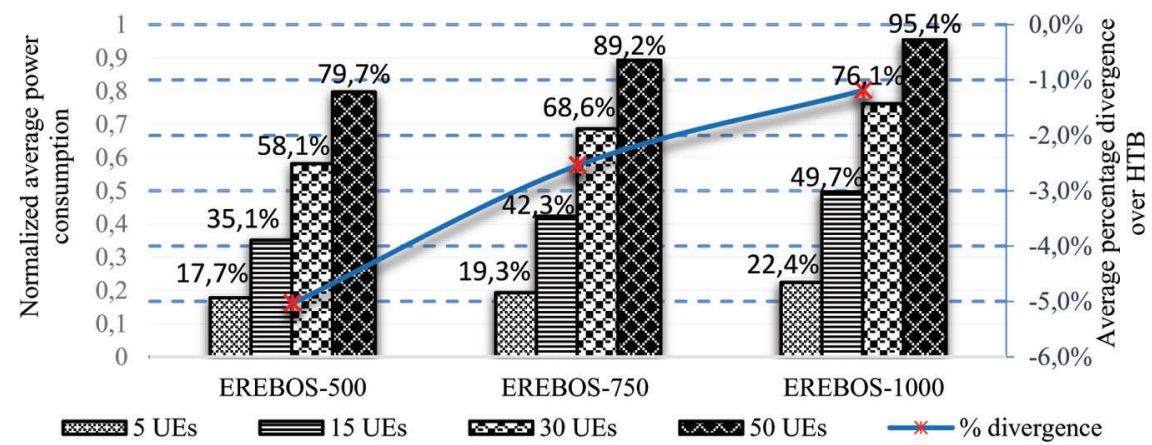

Figure 6 Normalized average power consumption against FOT and average percentage divergence among EREBOS's and HTBs' normalized average power consumption performances. 500,750 or 1000 stands for the GBR (in kbps) in each scenario applied.

the network operates with no energy efficient aware scheme, thus assuming that all BSs are operational.

The average power consumption of a FOT network's traffic load is calculated based on the average of all our simulation snapshots' runs, and it includes the sum of (i) the fixed power consumption cost of all BSs and (ii) the average power cost for access and backhaul transmissions, recorded during the evaluation of each algorithm (EREBOS or HTB). EREBOS achieves power consumption reduction in all examined scenarios against FOT and the reference HTB algorithm. We record an up to $82.3 \%$ power consumption reduction compared to FOT at the lowest network's traffic load conditions of 5 UEs with $500 \mathrm{kbps}$ as GBR. Yet, EREBOS performs by up to 
$5 \%$ better compared to the reference algorithm, in terms of normalized average power consumption, again at the same scenario of the lowest traffic load.

The transmission part of a network can operate more efficiently by careful utilization of its resources even when no BS activation strategy is applied. FOT networks' power consumption average metrics were calculated up to $6.7 \%$ lower when the EREBOS heuristic's metrics were utilized for estimating FOT network's power consumption, compared to the reference algorithm. EREBOS approach exhibits an energy consumption alleviation trend compared to the reference algorithm in all scenarios examined, as depicted in Figure 7.

The energy efficiency gains of the EREBOS over FOT is also depicted via the well-known bit per joule metric in Figure 8. EREBOS algorithm improves energy efficiency by up to $465 \%$ against FOT, where the biggest gains were recorded in lower network's traffic load conditions and outperforms the reference algorithm at all scenarios examined, achieving up to $~ 30 \%$ increase in the useful payload per Joule.

\subsection{Comparison of Resource Utilization}

EREBOS's higher utilization of the most efficient network's resources for serving the access links is responsible for the increased alleviation in the total network's energy consumption. Micro BSs resources' average utilization is linearly increased up to $\sim 320 \%$ compared to the reference algorithm followed by a reduction of the macro BSs resources' average utilization, whose energy consumption is much higher, by up to $\sim 38 \%$ at the lowest traffic load of 5 UEs examined, as is shown in Figure 9.

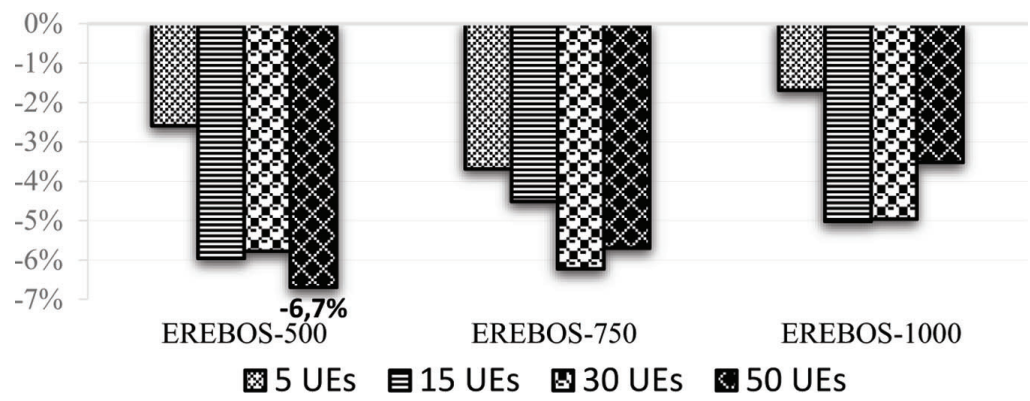

Figure 7 FOT network's average power consumption alleviation using as input the EREBOS's metrics compared to the HTB algorithm. 500, 750 or 1000 stands for the GBR (in kbps) in each scenario applied. 


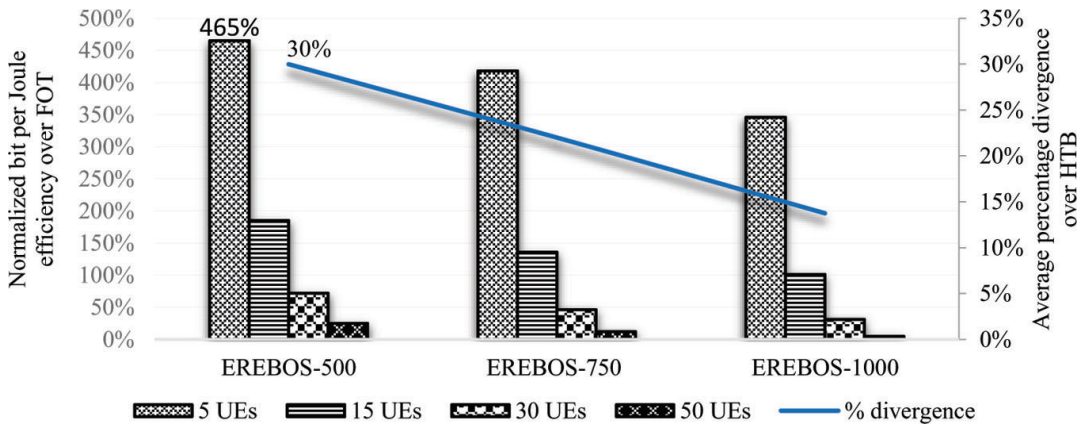

Figure 8 Normalized bit per Joule efficiency over FOT and the corresponding average divergence of EREBOS compared to HTB reference algorithm. 500, 750 or 1000 stands for the GBR (in kbps) in each scenario applied.

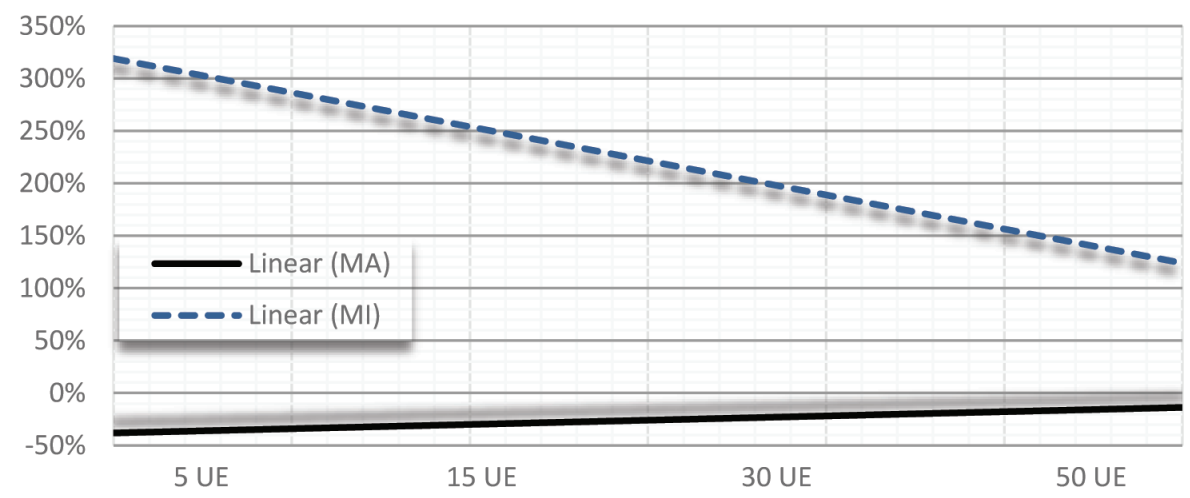

Figure 9 EREBOS's linear percentage representation of the network's average resources utilization for various traffic load scenarios compared to the reference algorithm. MI(MA) stands for Micro(Macro).

\subsection{Power Consumption Comparison in a Daily Operational Cycle}

The overall normalized power consumption and bit per joule percentage improvement over FOT, based on a real daily traffic [50] are presented in Figure 10. Overall, we could serve the daily telecommunication traffic in our network more efficiently, since according to our calculations the necessary energy for running this heterogeneous mobile network was reduced by $\sim 37 \%$, while providing $\sim 92.5 \%$ more information per Joule compared to FOT networks. EREBOS algorithm consumes $\sim 2.61 \%$ less and provides $\sim 6.2 \%$ more information per Joule compared to the reference algorithm. 


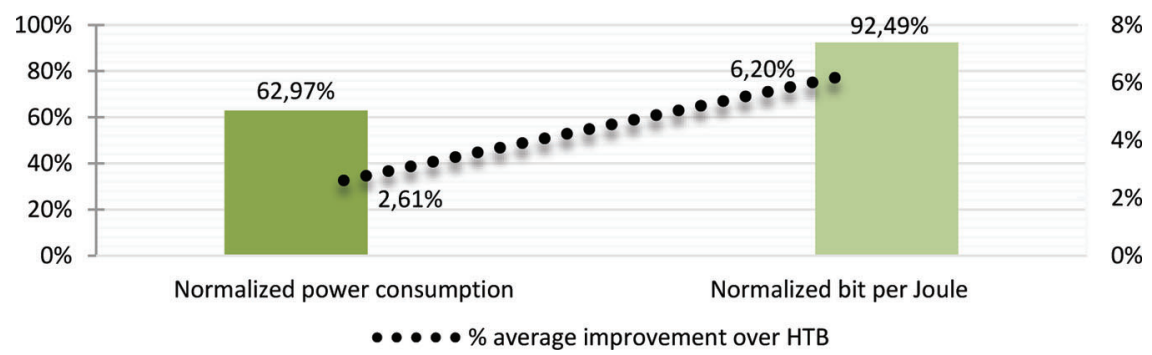

Figure 10 Overall normalized power consumption and bit per joule percentage improvement over FOT and over reference algorithm, based on a real daily traffic profile [50].

\subsection{Comparison of Computational Performance}

The computational performance of EREBOS, reference (HTB) and exhaustive algorithms is presented in Tables 2 and 3, where the average computational time of each algorithm for calculating the optimal solution is depicted. Each column entry represents time in seconds. In Table 2, we present the computational time of each algorithm running on the scaled down topology depicted in Figure 4. EREBOS computational time performance is superior and we can easily clarify the time-consuming process of estimating the optimal solution with the exhaustive search method; for the heaviest traffic load of 15 UEs, EREBOS calculates the optimal solution in 0,54 seconds, the reference algorithm in 0.76 seconds and exhaustive search in 244 seconds.

In Table 3, the computational time of EREBOS and HTB algorithms is presented, on the original heterogeneous mobile topology depicted in Figure 3. EREBOS computational performance again is superior compared to the reference algorithm. Both heuristic schemes remain rather immune to GBR increase, but both are affected from the traffic load (number of UEs) increase. This is in accordance with the formula of computational time presented at the end of Section 3, which includes the number I of UEs, but not the UEs' bitrate requirements.

Table 2 Computational time of EREBOS, HTB and exhaustive heuristics, while each one calculates the optimal solution in the smaller topology depicted in Figure 4, for GBR of $500 \mathrm{kbps}$. Time is in seconds

\begin{tabular}{ccccc}
\hline \# UEs & Heuristic & & & \\
& & EREBOS & HTB & EXHAUSTIVE \\
\hline 2 & 0,28 & 0,34 & 115,19 \\
5 & 0,36 & 0,46 & 157,66 \\
10 & 0,45 & 0,51 & 244,99 \\
15 & 0,54 & 0,76 & 244,01 \\
\hline
\end{tabular}


Table 3 Computational time of EREBOS and HTB heuristics in the original network topology depicted in Figure 3. Time is in seconds

\begin{tabular}{|c|c|c|c|c|c|c|}
\hline \multirow{2}{*}{ \# UEs } & \multicolumn{2}{|c|}{ GBR of $500 \mathrm{kbps}$} & \multicolumn{2}{|c|}{ GBR of $750 \mathrm{kbps}$} & \multicolumn{2}{|c|}{ GBR of $1000 \mathrm{kbps}$} \\
\hline & EREBOS & HTB & EREBOS & HTB & EREBOS & HTB \\
\hline 5 & 0,57 & 2,84 & 0,55 & 2,86 & 0,56 & 2,87 \\
\hline 15 & 1,19 & 4,51 & 1,09 & 4,65 & 1,11 & 4,43 \\
\hline 30 & 1,9 & 7,78 & 2,12 & 9,2 & 2,33 & 7,94 \\
\hline 50 & 3,83 & 14,55 & 3,93 & 13,25 & 3,72 & 13,23 \\
\hline
\end{tabular}

Concluding, EREBOS heuristic achieves great energy savings against FOT heterogeneous networks and outperforms the reference heuristic HTB, by properly utilizing the lower power consumption network's resources and effectively managing the necessary resources of our network to serve access and backhaul links under the same spectrum and carrier frequencies as used at the macro layer. The above results prove that EREBOS performs near optimally, especially at lower traffic conditions, within acceptable computational times.

\section{Conclusions}

In this paper, we provide a backhaul-aware energy optimization heuristic for heterogeneous mobile networks where wireless in-band backhaul is deployed as the data transmission method between macro BSs and operational micro BSs. The corresponding power consumption optimization problem is formulated. An exhaustive search method is applied to compare the performance of the heuristic proposed. Our approach proved to perform near optimally, especially on lower traffic load conditions. From the results obtained it is shown that the energy efficient heuristic proposed can alleviate by up to $\sim 82.3 \%$ the total power consumption against FOT. The application of the heuristic proposed may lead to total heterogeneous network's power consumption alleviation by up to $\sim 37 \%$ while useful information per Joule transmitted may be by up to $\sim 92.5 \%$ increased during their daily operational cycle, compared to similar FOT networks.

\section{References}

[1] Cisco (2017). Cisco Visual Networking Index: Global Mobile Data Traffic Forecast Update, 2015-2020. Available at: http://www.cisco.com/c/en/ us/solutions/collateral/service-provider/visual-networking-index-vni/ vni-forecast-qa.pdf 
[2] Cisco (2011). Cisco Visual Networking Index: Global Mobile Data Traffic Forecast Update, 2010-2015. Available at: https://engineering.nd.edu/ news-publications/pressreleases/Cisco_VNI_Global_Mobile_Data_ Traffic_Forecast_2010_2015.pdf

[3] Ericsson (2016). Ericsson Mobility Report 2016. Available at: https:// www.ericsson.com/res/docs/2016/ericsson-mobility-report-2016.pdf

[4] Li, B., Zhu, D., and Liang, P. (2014). Small cell in-band wireless backhaul in massive MIMO systems: a cooperation of next-generation techniques. IEEE Trans. Wireless Commun. 12, 7057-7069.

[5] Fehske, A. J., Richter, F., and Fettweis, G. P. (2009). "Energy efficiency improvements through micro sites in cellular mobile radio networks," in Proceedings of the 2009 IEEE Globecom Workshops, Honolulu, HI, 1-5. doi: 10.1109/GLOCOMW.2009.5360741

[6] Richter, F., Fehske, A. J., and Fettweis, G. P. (2009). "Energy Efficiency Aspects of Base Station Deployment Strategies for Cellular Networks," in Proceedings of the 2009 IEEE 70th Vehicular Technology Conference Fall (VTC 2009-Fall), Anchorage, AK, 1-5. doi: 10.1109/VETECF.2009.5379031

[7] Koutitas, G., Karousos, A., and Tassiulas, L. (2012). Deployment strategies and energy efficiency of cellular networks. IEEE Trans. Wirel. Commun. 11, 2552-2563. doi: 10.1109/TWC.2012.050112.111461

[8] Wu, Y., and Niu, Z. (2012). "Energy efficient base station deployment in green cellular networks with traffic variations," in Proceedings of the 2012 1st IEEE International Conference on Communications in China (ICCC), Beijing, 399-404. doi: 10.1109/ICCChina.2012.6356915

[9] Li, C., Zhang, J., and Letaief, K. (2014). Throughput and energy efficiency analysis of small cell networks with multi-antenna base stations. IEEE Trans. Wirel. Commun. 13, 2505-2517. doi: 10.1109/TWC.2014.031714.131020

[10] Gonzalez-Brevis, P., Gondzio, J., Fan, Y., Vincent Poor, H., Thompson, J., Krikidis, I., and Chung, P.-J. (2011). "Base Station Location Optimization for Minimal Energy Consumption in Wireless Networks," in Proceedings of the 2011 IEEE 73rd Vehicular Technology Conference (VTC Spring), Budapest, 1-5. doi: 10.1109/VETECS.2011.5956204

[11] Oh, E., Son, K., and Krishnamachari, B. (2013). Dynamic base station switching-on/off strategies for green cellular networks. IEEE Trans. Wirel. Commun. 12, 2126-2136. doi: 10.1109/TWC.2013.032013 .120494 
[12] Guha, S., Chau, C. K., and Basu, P. (2010). "Green wave: latency and capacity-efficient sleep scheduling for wireless networks," in Proceedings of the 2010 IEEE INFOCOM, San Diego, CA, 1-9. doi: 10.1109/INFCOM.2010.5462011

[13] Saker, L., Elayoubi, S. E., Combes, R., and Chahed, T. (2012). Optimal control of wake up mechanisms of femtocells in heterogeneous networks. IEEE J. Sel. Areas Commun. 30, 664-672. doi: 10.1109/JSAC.2012.120415

[14] Niu, Z., Wu, Y., Gong, J., and Yang, Z. (2010). Cell zooming for costefficient green cellular networks. IEEE Commun. Mag. 48, 74-79. doi: 10.1109/MCOM.2010.5621970

[15] Micallef, G., Mogensen, P., and Scheck, H. O. (2010). "Cell size breathing and possibilities to introduce cell sleep mode," Proceedings of the Wireless Conference (EW), 2010 European, Lucca, 111-115. doi: 10.1109/EW.2010.5483401

[16] Wang, C. Y., Ko, C. H., Wei, H. Y., and Vasilakos, A. V. (2016). A VotingBased Femtocell Downlink Cell-Breathing Control Mechanism. IEEE ACM Trans. Netw. 24, 85-98. doi: 10.1109/TNET.2014.2357498

[17] Suarez, L., Nuaymi, L., and Bonnin, J. M. (2012). "Analysis of the overall energy savings achieved by green cell-breathing mechanisms," in Proceedings of the Sustainable Internet and ICT for Sustainability (SustainIT), Pisa, 1-6.

[18] Suarez, L., and Nuaymi, L. (2015). "Multi-size cell expansion for energyefficient cell breathing in green wireless Networks," in Proceedings of the Vehicular Technology Conference (VTC Fall), 2015 IEEE 82nd, Boston, MA, 1-5. doi: 10.1109/VTCFall.2015.7391031

[19] Soh, Y. S., Quek, T. Q. S., Kountouris, M., and Shin, H. (2013). Energy efficient heterogeneous cellular networks. IEEE J. Sel. Areas Commun. 31, 840-850. doi: 10.1109/JSAC.2013.130503

[20] Kyriazis, G. Rouskas, A., and Karetsos, G. T. (2015). "Energy-efficient base station management in heterogeneous networking environments," in Proceedings of the 2015 IEEE Symposium on Computers and Communication (ISCC), Larnaca, 283-288, doi: 10.1109/ISCC.2015.7405529

[21] Kyriazis, G. and Rouskas, A. (2016). Design and operation of energy efficient heterogeneous mobile networks. Wirel. Netw. 22, 2013-2028. doi: 10.1007/s11276-015-1083-0

[22] Yang, Y., Chen, L., and Wang, W. (2013). "A novel energy saving scheme based on base stations dynamic configuration in green cellular networks," 
in Proceedings of the 2013 IEEE 78th Vehicular Technology Conference (VTC Fall), Las Vegas, NV, 1-5. doi: 10.1109/VTCFall.2013.6692051

[23] Lopes, A. R. M. Farias, F. S. and Costa, J. C. W. A. (2015). Evaluation of the energy impact on indoor small cells and backhaul. Int. J. Future Comput. Commun. 4, 152-159.

[24] Tombaz, S., Zheng Z., and Zander, J. (2013). "Energy efficiency assessment of wireless access networks utilizing indoor base stations," in Proceedings of the 2013 IEEE 24th International Symposium Personal Indoor and Mobile Radio Communications (PIMRC), London, 3105-3110, doi: 10.1109/PIMRC.2013.6666680

[25] Tombaz, S., Monti, P., Farias, F., Fiorani, M., Wosinska, L., and Zander, J. (2014). "Is backhaul becoming a bottleneck for green wireless access networks?," in Proceedings of the 2014 IEEE International Conference on Communications (ICC), Sydney, NSW, 4029-4035. doi: 10.1109/ICC.2014.6883951

[26] Barth, U. (2009). Alcatel-Lucent, Bell Labs Stuttgart, How To ReduceGreen House Gas Emissions From ICT Equipment, (Slides) Wireless Networks, EARTH Research Project, ETSI Green Agenda. Valbonne: ETSI.

[27] Mesodiakaki, A., Adelantado, F., Antonopoulos, A., Kartsakli, E., Alonso, L., and Verikoukis, C. (2014). "Energy impact of outdoor small cell backhaul in green heterogeneous networks," in Proceedings of the 2014 IEEE 19th International Workshop on Computer Aided Modeling and Design of Communication Links and Networks (CAMAD), Athens, 11-15, doi: 10.1109/CAMAD.2014.7033196

[28] Ge, X., Cheng, H., Guizani, M., and Han, T. (2014). 5G wireless backhaul networks: Challenges and research advances. IEEE Netw. 28, 6-11.

[29] Tombaz, S., Monti, P., Wang, K., Vastberg, A., Forzati, M., and Zander, J. (2011). "Impact of backhauling power consumption on the deployment of heterogeneous mobile networks," in Proceedings of the 2011 IEEE Global Telecommunications Conference (GLOBECOM 2011), Houston, TX, 1-5. doi: 10.1109/GLOCOM.2011.6133999

[30] Suárez, L., Bouraoui, M. A., Mertah, M. A., Morvan, M., and Nuaymi, L. (2015). Energy efficiency and cost issues in backhaul architectures for high data-rate green mobile heterogeneous networks, in Proceedings of the 2015 IEEE 26th Annual International Symposium on Personal, Indoor, and Mobile Radio Communications (PIMRC), Hong Kong, 1563-1568. doi: 10.1109/PIMRC.2015.7343547 
[31] Aleksic, S., Deruyck, M., Vereecken, W., Joseph, W., Pickavet, M., and Martens, L. (2013). Energy efficiency of femtocell deployment in combined wireless/optical access networks. Comput. Netw. 57, 1217-1233. doi: 10.1016/j.comnet.2012.12.013

[32] Mahloo, M., Monti, P., Chen, J., and Wosinska, L. (2014). "Cost modeling of backhaul for mobile networks," in Proceeding of the 2014 IEEE International Conference on Communications Workshops (ICC), Sydney, NSW, 397-402. doi: 10.1109/ICCW.2014.6881230

[33] Yang, H. H., Geraci, G., and Quek, T. Q. S. (2016). Energy-efficient design of MIMO heterogeneous networks with wireless backhaul. IEEE Trans. Wireless Commun. 15, 4914-4927. doi: 10.1109/TWC.2016.2549529

[34] 3GPP (2010). Overview of 3GPP Release 10. Available at: http:// www.3gpp.org/specifications/releases/70-release-10

[35] Radio Spectrum Policy Group (2015). RSPG15-607: RSPG Report on Spectrum Issues on Wireless Backhaul. Brussels: European Commission.

[36] Sharma, A., Ganti, R. K., and Milleth, J. K. (2016). "Performance analysis of full duplex self-backhauling cellular network," in Proceedings of the 2016 IEEE International Conference on Communications (ICC), Kuala Lumpur, 1-6. doi: 10.1109/ICC.2016.7511121

[37] Sharma, A., Ganti, R. K., and Milleth, J. K. (2017). Joint backhaul-access analysis of full duplex self-backhauling heterogeneous networks. IEEE Trans. Wirel. Commun. 16, 1727-1740. doi: 10.1109/TWC.2017.2653108

[38] Cabrera-Mercader, C., Chen, L.-W., Fallik, B., Hu, S., and Chapin, J. (2007). In-Band Wireless Backhaul using SDR for Rural Cellual Systems. Cambridge, MA: Vanu, Inc.

[39] Coletti, C., Mogensen, P., and Irmer, R. (2011). "Deployment of LTE inband relay and micro base stations in a realistic metropolitan scenario," in Proceedings of the Vehicular Technology Conference (VTC Fall), San Francisco, CA, 1-5. doi: 10.1109/VETECF.2011.6093073

[40] Coletti, C., Mogensen, P., and Irmer, R. (2011). "Performance analysis of relays in LTE for a realistic suburban deployment scenario," in Proceedings of the 2011 IEEE 73rd Vehicular Technology Conference (VTC Spring), Budapest, 1-5. doi: 10.1109/VETECS.2011.5956245

[41] Li, B., Zhu, D., and Liang, P. (2015). Small cell in-band wireless backhaul in massive MIMO systems: a cooperation of nextgeneration techniques. IEEE Trans. Wirel. Commun. 14, 7057-7069. doi: 10.1109/TWC.2015.2464299 
[42] Auer, G., Giannini, V., Desset, C., Gódor, I., Skillermark, P., Olsson, M., et al. (2011). How much energy is needed to run a wireless network? IEEE Wirel. Commun. 18, 40-49. doi: 10.1109/MWC.2011.6056691

[43] Sridharan, R., (1995). The capacitated plant location problem. Eur. J. Oper. Res.87, 203-213.

[44] Prasad, A., and Maeder, A. (2015). Backhaul-aware energy efficient heterogeneous networks with dual connectivity. Telecommun. Syst. 59:25. doi:10.1007/s11235-014-9893-4

[45] Kyriazis, G., and Rouskas, A. (2016). "Energy efficient wireless inband backhaul in heterogeneous networking environments," in Proceedings of the 2016 24th International Conference on Software, Telecommunications and Computer Networks (SoftCOM), Split, 1-6. doi: 10.1109/SOFTCOM.2016.7772138

[46] Hentilä, L., Kyösti, P., Käske, M., Narandzic, M., and Alatossava, M. (2007). MATLAB implementation of the WINNER Phase II channel model ver1.1. Available at: http://www.ist-winner.org/pha se2model.html

[47] Bublin, M., Hardouin, E., Hrdlicka, O., Kambourov, I., Legouable, R., Olsson, M., et al. (2007). Simon Plass, IST-4-027756 WINNER II D4.7.1 v.1.0 Interference Averaging concepts. Available at: http://projects.celticinitiative.org/winner+/WINNER2-Deliverables/D4.7.1.pdf

[48] Alcatel-Lucent and Vodafone Chair on Mobile Communication Systems. (2009). Study on Energy Efficient Radio Access Network (EERAN) Technologies. Dresden: Technical University of Dresden (Unpublished Project Report).

[49] 3GPP TR 36.814 V9.0.0 (2010-2013). Specification. Available at: http://www.qtc.jp/3GPP/Specs/36814-900.pdf

[50] EARTH (2013). EARTH Project. Available at: https://www.ict-earth.eu/ 


\section{Biographies}

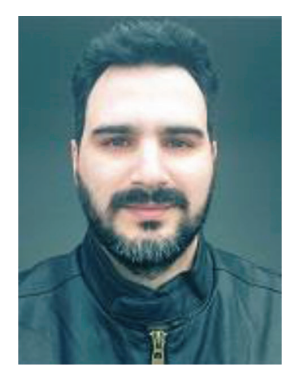

G. Kyriazis is a Ph.D. candidate in the Department of Digital Systems of the School of Information and Communication Technologies at the University of Piraeus, GREECE, since 2011. He has also received his BS and MS degrees in 2006 and 2009 respectively from the same department. Mr Kyriazis has participated (under scholarship) in the "THALES - ENERGY EFFICIENT DESIGN OF COMMUNICATIONS NETWORKS" EU founded project (09/2013-02/2015), being responsible for designing and developing of energy efficient wireless mobile cognitive networks' solutions and worked for 3 years in Siemens Hellas, as a Software Engineer on landline telecommunication networks (2007-2010). His main research focus is on energy efficient mobile networks planning and optimization.

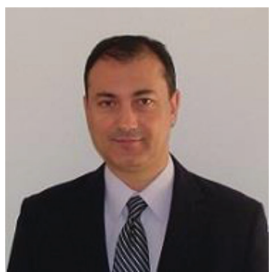

A. Rouskas received the five-year Diploma in electrical engineering from the National Technical University of Athens (NTUA), the MS degree in communications and signal processing from Imperial College, London, and the Ph.D. degree in electrical and computer engineering from NTUA. He is currently an Associate Professor in the Department of Digital Systems, University of Piraeus, Greece, and has been a Lecturer, Assistant Professor and Director of the Computer and Communication Systems Laboratory in the Department Information and Communication Systems Engineering, University of the Aegean, Greece (2000-2009). During the last decade 
Dr Rouskas has been teaching many undergraduate and postgraduate courses on wireless networks, mobile communications and computer networking and has been involved in several Greek and European funded research projects, while in the past has been a member of the Network Design Department of the Greek Cellular Operator CosmOTE. Dr Rouskas was the TPC co-chair of the European Wireless 2006 Conference and has served as a TPC member in several international conferences. His main research interests are in the areas of next generation mobile and wireless communication networks. 
